\title{
AVALIAÇÃo DE UM NOVO REATOR DE CAVITAÇÃO PARA A PRODUÇÃO DE EMULSÕES NANOMÉTRICAS
}

\author{
M. G. R. SANTOS ${ }^{1}$, M. M. E. COLMÁN ${ }^{1}$, C. R. OLIVEIRA ${ }^{1}$ e R. GIUDICI ${ }^{1}$ \\ ${ }^{1}$ Universidade de São Paulo - USP, Escola Politécnica, Departamento de Engenharia Química \\ Email para contato: marcelogiudice@usp.br
}

\begin{abstract}
RESUMO - O presente trabalho consiste na avaliação de um novo modelo de reator de cavitação hidrodinâmica para a produção de emulsões nanométricas de óleo em água. Foi estudada a eficiência desse tipo de reator em produzir emulsões nanométricas de vaselina industrial em água na proporção volumétrica de $12 \%$ (O/A). Como surfactante utilizou-se Lauril Sulfato de Sódio. Os testes foram realizados em reciclo e amostras foram coletadas em intervalos fixos de tempo para posterior análise. As emulsões foram, então, analisadas quanto à distribuição de tamanho de gotas e estabilidade, permitindo constatar a eficiência desse tipo de equipamento para a produção em escala industrial dessas emulsões. Foi possível atingir tamanhos de gotas de óleo de $590 \mathrm{~nm}$ e que permaneceram estáveis a um período superior a quatro semanas.
\end{abstract}

\section{INTRODUÇÃO}

Os métodos para a produção de emulsões em escala nanométrica são, usualmente, classificados como sendo de alta energia, baixa energia ou uma combinação de ambos. Tratando exclusivamente dos métodos de alta energia, esses são divididos em classes de acordo com o princípio utilizado, a saber: HSH (High Speed Homogeneizers), HPH (High Pressure Homogeneizers), Microfluidizer e Ultrasom . Por serem intensivos em energia, a utilização em escala industrial ainda representa um desafio à engenheiros e pesquisadores (Koroleva e Yurtov, 2012).

Dentre os métodos de alta energia, aqueles que encontram maior potencial de serem aplicados em escala industrial são os do tipo HSH (High Speed Homogeneizers), Microfludizer e HPH (High Pressure Homogeneizers) (Schutz et al, 2004). Nesse tipo de equipamento, a mistura óleo/água é submetida à condições extremas de escoamento, permitindo a ocorrência de forças de cisalhamento, turbulência e cavitação. Acerca do fenômeno da cavitação é interessante constar a sua importância dentre os processos de emulsificação de alta energia (Gogate e Pandit, 2001).

Equipamentos do tipo HSH tem como princípio de funcionamento a presença de uma parte estacionária e outra móvel, respectivamente estator e rotor. Quando o rotor atinge velocidades críticas, a pressão local periférica cai abaixo da pressão de vapor, desencadeando a nucleação e implosão de bolhas de vapor - fenômeno da cavitação. Outros efeitos importantes nesse tipo de equipamento e que contribuem para o processo de emulsificação são: turbulência, forças de cisalhamento e oscilações de pressão (Senthilkumar e Pandit, 1999). 
Emulsões estão presentes no dia a dia, em quase a maioria dos produtos consumidos, sejam alimentos, personal care, produtos farmacêuticos, utilidades para a indústria de construção como tintas, produção de vernizes e colas. As emulsões podem ser definidas como uma dispersão coloidal de dois líquidos imiscíveis. O qual o tamanho das gotas da fase dispersa podem se encontrar na escala de nanômetros e, em alguns casos, em micrometros. Para a obtenção de emulsões estáveis é necessário utilização de um terceiro componente chamado de agente emulsificante e ou surfactante (Morrison e Ross, 2002). Para garantir a estabilidade da emulsão deve ser levada em consideração a natureza do surfactante, de ambas as fases e a relação da quantidade de cada um dos componentes. (Leal-Calderon, et al., 2007).

Neste trabalho foi avaliada a eficiência de um novo modelo de reator de cavitação hidrodinâmica, do tipo HSH (High Speed Homogeneizers), para a produção de emulsões O/A em escala nanométrica. Foram estudadas as influências da rotação imposta ao rotor e da quantidade de surfactante utilizada no diâmetro médio de partículas e na estabilidade das emulsões produzidas. $\mathrm{O}$ equipamento também foi avaliado quanto à eficiência energética.

\section{MATERIAIS E MÉTODOS}

Neste trabalho, utilizou-se um reator de cavitação do tipo HSH (sob processo de patente), que opera acoplado a um motor elétrico trifásico, $220 \mathrm{~V}$, de $18.5 \mathrm{~kW}$ de potência, comandado por um inversor de frequência para o controle da rotação imposta ao rotor. Ambos, rotor e estator, na forma de discos com ranhuras em baixo relevo, possuem rebaixos idênticos uniformemente espaçados e distribuídos ao longo dos perímetros. O reator foi confeccionado em escala industrial, sendo as características operacionais para a produção de emulsões apresentadas na Tabela 1:

Tabela - 1. Características Operacionais

\begin{tabular}{|c|c|c|}
\hline & Valor Mínimo & Valor Máximo \\
\hline Vazão (L/min) & 12 & 20 \\
\hline Rotação do Rotor (RPM) & 2000 & 2500 \\
\hline
\end{tabular}

Para a realização dos testes utilizou-se um circuito fechado contendo o reator (HSH), medidores em linha (vazão, temperatura e pressão) e três tanques para a realização de ensaios em reciclo, conforme mostra a Figura 1. 


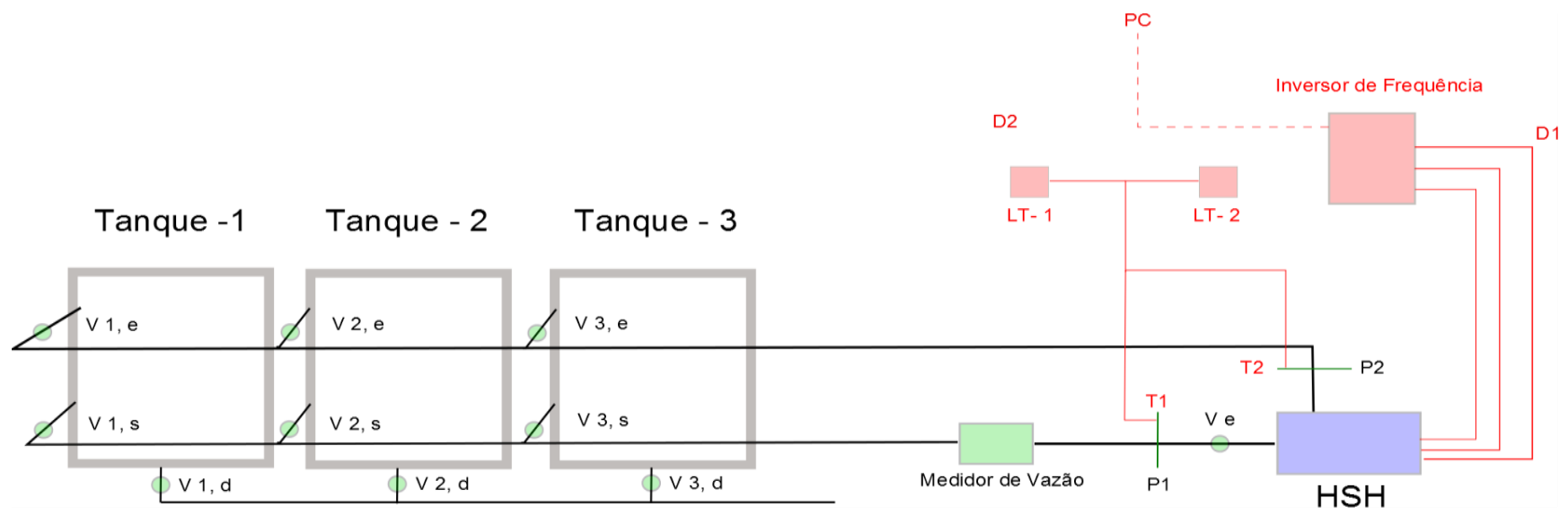

Figura 1 - Esquema da planta de testes (V - válvulas , T - termopares, P - manômetros, LT Displays para leitura da temperatura, $\mathrm{HSH}$ - reator de cavitação hidrodinâmica do tipo rotorestator comandado pelo inversor de frequência).

\subsection{Ensaios De Emulsificação}

Foi estudada a produção de uma emulsão O/A com a seguinte composição, conforme apresentado na Tabela 2. O surfactante utilizado foi o Lauril Sulfato de Sódio (SLS) e a água MiliQ conformaram a fase contínua. O óleo mineral, da Marca Hellman, foi utilizado como fase dispersa.

Tabela 2. Composição da Emulsão Estudada

\begin{tabular}{|c|c|c|}
\hline & Substância & Volume (L) \\
\hline Fase Contínua & Água Mili-Q & 45 \\
\hline Fase Dispersa & Vaselina Líquida & 6 \\
\hline
\end{tabular}

Preparo da mistura: São adicionados a um dos tanques o volume de 45 litros de água Mili-Q e a quantidade de surfactante. $\mathrm{O}$ volume é homogeneizado por agitação manual. Após esse preparo o reator de cavitação, HSH, é ligado e mantido a uma rotação fixa de 850 RPM, o volume do tanque é, então, colocado em reciclo passando pelo HSH e retornando ao mesmo tanque. Após 6 minutos de processamento a fase oleosa é adicionada ao tanque. A mistura é então deixada em reciclo por mais 6 minutos. Essa etapa é importante para permitir uma mistura prévia e homogeneização do conteúdo.

Emulsificação em reciclo: Após a etapa de preparação faz-se a configuração da velocidade de rotação deseja através do inversor de frequência. Essa rotação é mantida fixa até o final do ensaio. O volume do tanque é, então, continuamente processado a uma vazão constante, passando pelo reator de cavitação e retornando ao tanque. As amostras foram coletadas em intervalos de cinco minutos até 
aproximadamente 30 minutos. Este tempo de 30 minutos foi estipulado devido ao aumento da temperatura da emulsão durante a homogeneização, a qual foi continuamente ao longo do processo. $\mathrm{O}$ ponto de coleta dessas amostras é na saída do HSH.

Caracterização das emulsões: O tamanho médio das gotas foi determinado por meio do equipamento Beckman Coulter, Coulter N4 Plus (média e intensidade), o qual utiliza a técnica de espalhamento dinâmico de luz. A preparação das amostras para análise do diâmetro de gotas foi diluída com água destilada de acordo a concentração adequada à faixa de leitura do equipamento. Para a avaliação da estabilidade das emulsões produzidas optou-se por analisar a última amostra de cada teste em um equipamento do tipo Turbiscan por um período de 4 horas à temperatura ambiente. Além deste, a emulsão produzida após o ensaio foi deixada em repouso no tanque por 24 horas, após o qual uma nova amostra foi coletada para uma reavaliação do tamanho médio de gotas no equipamento Coulter N4 Plus.

Para cada ensaio realizado foi montada uma tabela contendo a vazão medida no momento da coleta (a qual procurou-se manter a mais fixa e estável possível), o tempo transcorrido após o início da etapa de emulsificação, as temperaturas de entrada e saída do reator ( T1 e T2, respectivamente), o diâmetro médio de partícula (DP) e o respectivo índice de polidispersividade (PDI), conforme pode ser visto na Tabela 3 a seguir.

Tabela 3 - Tabela de Resultados

\begin{tabular}{|c|c|c|c|c|c|}
\hline Vazão (L/min ) & Tempo (min) & DP $(\mathrm{nm})$ & PDI & T1 $\left({ }^{\circ} \mathrm{C}\right)$ & T2 $\left({ }^{\circ} \mathrm{C}\right)$ \\
\hline & & & & & \\
\hline
\end{tabular}

\section{DISCUSSÃO DE RESULTADOS}

Após a realização de ensaios preliminares foi possível constatar a existência de uma faixa operacional ideal para o processo de emulsificação, onde é possível obter um menor tamanho médio de partículas e maior estabilidade. Essas condições ótimas situam-se entre 2000 e 2500 RPM e trabalhando com uma vazão média de $17 \mathrm{~L} / \mathrm{min}$.

\subsection{Efeito da Rotação}

O gráfico a seguir compara as emulsões produzidas pelo equipamento em 2000 e 2500 RPM. Ambos os ensaios foram realizados a uma vazão fixa de $17 \mathrm{~L} / \mathrm{min}$, nas proporções definidas pela Tabela 2 e utilizando 270,95 gramas de SLS. Os resultados dos ensaios a 2000 RPM e 2500 RPM foram plotados e interpolados por funções de potência, constatando a redução do tamanho médio de partícula em função do tempo de processamento. 


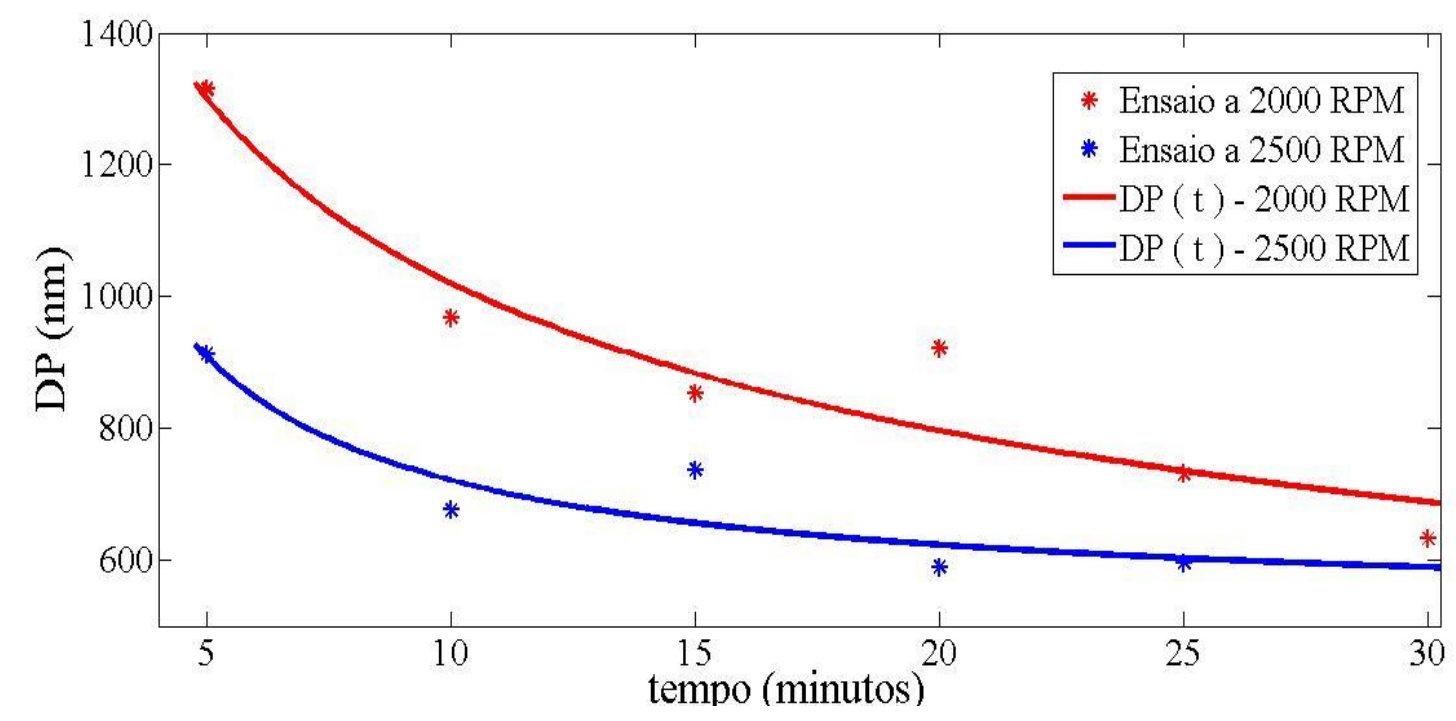

Figura 2 - Ensaios a 2000 e 2500 RPM

A partir dos resultados apresentados no gráfico da Figura 2 vê-se que um aumento na rotação permite com que emulsões mais finas sejam produzidas. Esse efeito é evidenciado pelo deslocamento para baixo da curva interpoladora à medida com que se aumenta a rotação imposta ao rotor do HSH .ou seja, partículas de menor tamanho são produzidas para o mesmo tempo de processo. A redução do tamanho médio de partícula deve-se à intensificação das condições de escoamento decorrente do aumento da rotação. É de se esperar que com a elevação da rotação haja um aumento das forças cisalhantes, turbulência, cavitação e pulsos de pressão e velocidade no líquido (Promtov, 2001), intensificando o processo de rompimento das gotas e criando emulsões mais finas, indicando que o aumento de energia no meio pode ser compensado por uma redução de tempo para atingir diâmetros menores.

$\mathrm{Na}$ literatura podem ser encontrados alguns trabalhos utilizando dispositivos em escala laboratorial como Ultra-som e Ultra Turrax que coincidem com os resultados deste trabalho (Maali e Mosavia, 2014) e (Koroleva e Yurtov, 2012).

Outra conclusão dos ensaios é quanto a existência de um limite mínimo de tamanho de partícula, abaixo do qual o equipamento não conseguiu reduzir mais o diâmetro médio. Esse limite situa-se entre 540 e $600 \mathrm{~nm}$. Importante constar que o limite somente foi confirmado para a emulsão $\mathrm{O} / \mathrm{A}$ com as características de acordo com a Tabela 1.

Conforme mencionado anteriormente, a estabilidade da emulsão foi avaliada utilizando um equipamento do tipo Turbiscan. O gráfico da Figura 3 a seguir é o resultado do ensaio a 2500 RPM, onde vê-se que a emulsão permaneceu consideravelmente estável por um período de 4 horas. 


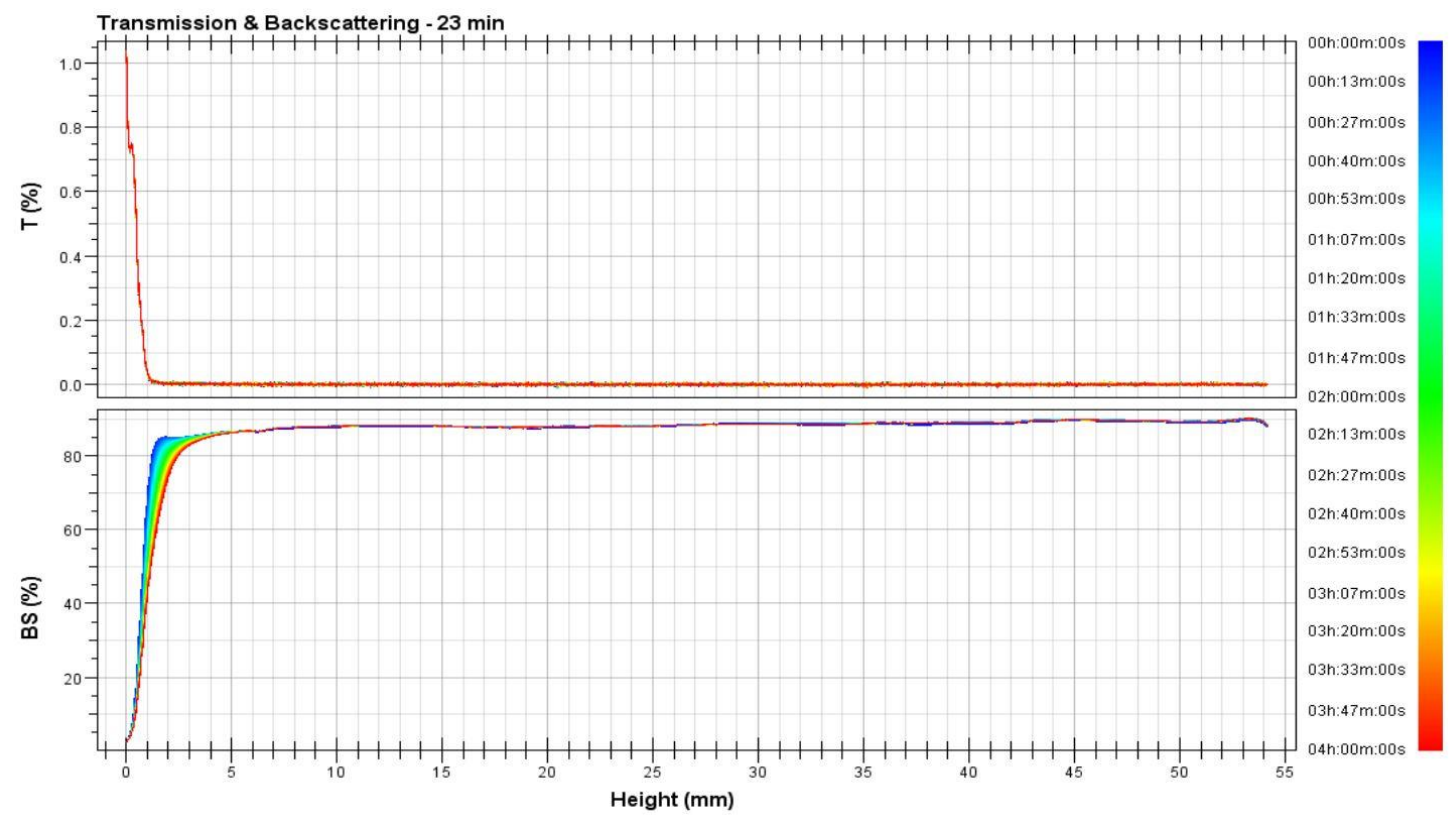

Figura 3. Resultado Turbiscan - Teste a 2500 RPM

Ainda sobre a estabilidade da emulsão, uma amostra de cada teste foi medida no equipamento Counter N4 após 24 horas do término do ensaio, não havendo alteração significativa no tamanho médio de partículas, conforme resultados da Tabela 4.

Tabela 4. Ensaios de tamanho de partículas

\begin{tabular}{|c|c|c|c|c|}
\hline & \multicolumn{2}{|c|}{ Medição após o ensaio } & \multicolumn{2}{c|}{ Medição após 24 horas do ensaio } \\
\hline & DP (nm) & PDI & DP (nm) & PDI \\
\hline 2000 & 632,4 & 0,479 & 592,1 & 0,369 \\
\hline 2500 & 595,5 & 0,447 & 627,2 & 0,288 \\
\hline
\end{tabular}

\subsection{Efeito da Quantidade de Surfactante}

A Figura 4 mostra o resultado de dois testes feitos às mesmas condições operacionais, diferenciando somente a quantidade de SLS utilizada. Ambos os testes foram realizados a 2500 RPM, e a uma vazão constante de $17 \mathrm{~L} / \mathrm{min}$. Em um dos ensaios utilizou-se 216,58 gramas de SLS, o que equivale à um teor de sólidos de 11,62\%, e no outro 270,93 gramas, ,71\% de teor de sólidos. A interpolação dos dados foi feita utilizando funções de potência. De onde é possível perceber que uma redução de $20 \%$ na quantidade de SLS resultou em uma emulsão com tamanho de partículas médio maior, o que é representado pelo deslocamento para cima da curva DP (nm) vs tempo (min). 


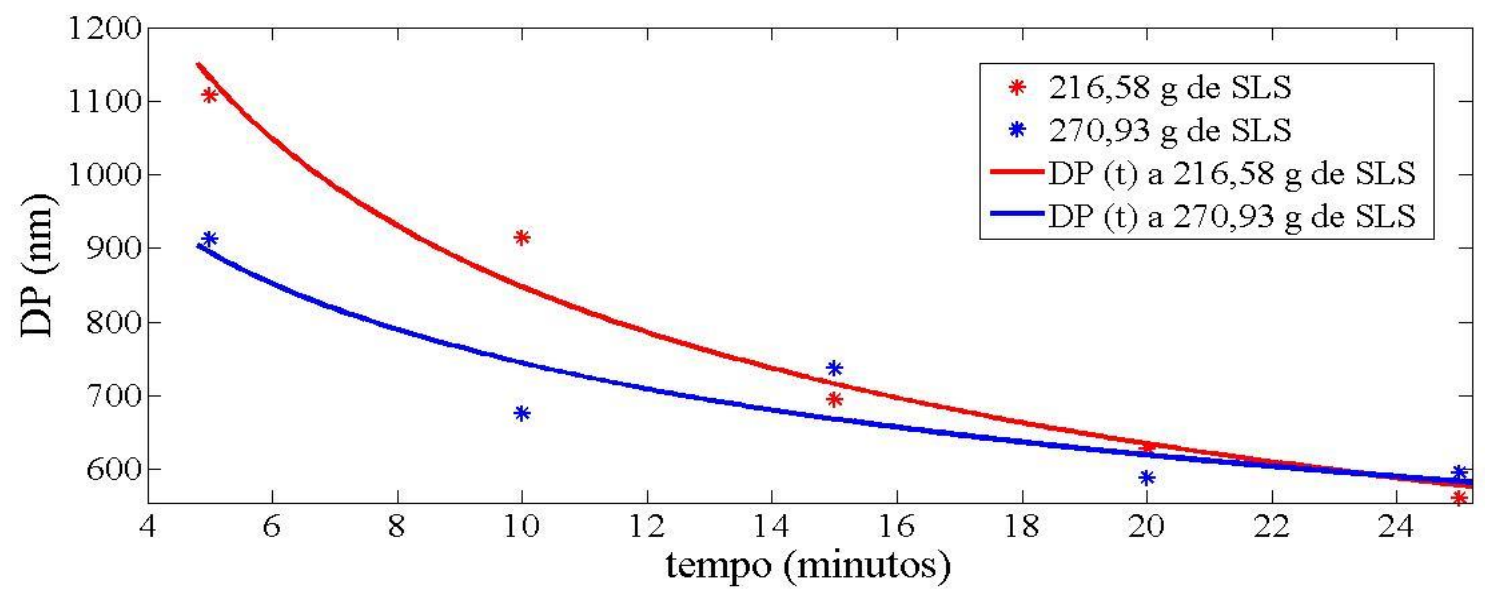

Figura 4. Diâmetro de partícula para diferentes teores de SLS

\subsection{Eficiência Energética}

Uma vez que o reator de cavitação estudado opera em uma faixa de vazão que pode ser interessante à processos industriais é interessante quantificar sua eficiência energética. Analisando a potência útil entregue pelo motor $\left(P_{u}\right)$ em função dos parâmetros operacionais, vazão $(v)$ e rotação (Rot), é possível definir uma relação linear entre essas variáveis, conforme a Equação 5 a seguir (Santos e Giudici, 2013):

$$
P_{u}(\operatorname{Rot}, v)=-24,63+0,01125 \cdot \operatorname{Rot}+0,5712 \cdot v \quad[k W]
$$

Tomando como exemplo o teste a 2500 RPM indicado na Figura 2, calculou-se a potência útil entregue pelo motor como sendo de $13,20 \mathrm{~kW}$ e o consumo de energia de 6,60 kWh. Nessas bases, o novo reator de cavitação, estudado neste trabalho, consumiria cerca de $25 \%$ da energia consumida por um método baseado em ultrassom (Abismail et al, 2000).

\section{CONCLUSÕES}

Com os resultados desse estudo foi possível constatar a eficiência desse novo modelo de reator de cavitação, do tipo HSH (High Speed Homogeneizer) para a produção de emulsões em escala nanométrica, tendo sido formuladas emulsões estáveis de tamanho médio de partículas da ordem de $590 \mathrm{~nm}$. O equipamento opera em faixa industrial, com capacidade produtiva da ordem de 1,02 m/h. O trabalho também foi conclusivo quanto ao efeito da rotação sobre o tamanho médio de partículas, onde foi possível constatar que um aumento da rotação implica na obtenção de partículas menores em um espaço de tempo menor. Apesar deste efeito, ficou claro que, após 30 minutos de operação em reciclo, as operações tanto a 2000 quanto a 2500 resultam na produção de emulsões estáveis e com 
tamanho médio de partículas similar e próximo a $600 \mathrm{~nm}$. O efeito da quantidade de tensoativo também foi estudado, concluindo que uma redução na quantidade de SLS adicionado resulta na geração de emulsões ligeiramente mais grosseiras.

As emulsões produzidas demonstram estabilidade satisfatória, permanecendo estáveis por períodos superiores a um mês. O equipamento possui potencial para futuras aplicações em escala industrial, permitindo a produção de $1 \mathrm{~m}^{3} / \mathrm{h}$ de emulsão ás custas de 13,20 kWh de energia.

\section{REFERÊNCIAS}

Abisma1l B.; Canselier, J.P.; Wilhelm, A.M.; Delmas, H.; Gourdon C. Emulsification processes: online study by multiple light scattering measurements. Ultrasonics Sonochemistry v.7, p 187-192, 2000

Gogate, P.; Pandit, B. A., Hydrodynamic Cavitation Reactors: A State of the Art Review. Reviews in Chemical Engineering, v.17, p 1-85, 2001.

Koroleva, M.Y.; Yurtov, E. V. Nanoemulsions: the properties, methods of preparation and promising applications. Russian Chemical Reviews., v. 81, p. 21-43, 2012.

Leal-Calderon, F., Shmitt, V., Bibette, J. Emulsion Science. Basic Principles. Ney York: Springer Science Bussines Media, LLC, 2007.

Maali, A.; Mosavian H. M. T. Preparation and Application of Nanoemulsions in the Last Decade (2000-2010). Journal of Dispersion Science and Technology., v. 34, p 92-105, 2013.

Morrison, I. D., Ross, S. Colloidal Dispersion. New York: Jonh Wiley and Sons, Inc., 2002.

Promtov, M. A., Hydroacoustic Emulsification in a Rotary Pulsed Cavitation Apparatus, Theoretical Foundations of Chemical Engineering, v. 35, p. 309-3011, 2001

Santos, M. d. G. R; Giudici, R. Estudo da Eficiência Energética de Reator de Cavitação Hidrodinâmica para o Aquecimento Controlado de Líquidos, Anais do VII Congresso Brasileiro de Termodinâmica Aplicada, 2013.

Senthilkumar, P.; Pandit, A. B., Modelling Hydrodynamic Cavitation, Chem. Eng. Technol., v. 22, p12, 1999.

Schultz, S.; Wagner, G.; Urban, K.;Ulrich, J. High Pressure Homogenization as a Process for Emulsion Formation. Chem. Eng. Technol. v. 27, p. 361-368, 2004

Tang, S. Y.; Syvakumar, M. A Novel and Facile Liquid Whistle Hydrodynamic Cavitation Reactor to Produce Submicron Multiple Emulsions. AIChE Journal, v. 59, p. 155-167, 2012. 